\title{
Dielectric Properties and Spatial Distribution of Polarization in Polyethylene Aged under ac Voltage in a Humid Atmosphere
}

\author{
M. Wübbenhorst, \\ Department of Polymer Technology, Delft University of Technology, Delft, Netherlands \\ J. Hornsby, M. Stachen, D. K. Das-Gupta \\ School of Electronic Engineering and Computer Systems, University of Wales, Bangor, UK \\ A. Bulinski, and $\mathrm{S}$. Bamji \\ National Research Council Canada, Ottawa, Canada
}

\begin{abstract}
This paper reports the results of an investigation of the dielectric properties, growth of water tree density and spatial distribution of polarization in samples of non-stabilized LDPE. Samples were initially sand-blasted to accelerate initiation of water trees, and subsequently aged in salt solution for approximately three weeks under two regimes of temperature (room temperature and $65^{\circ} \mathrm{C}$ ). Aging both with and without the presence of electric field stress was studied. The capacitance and dielectric loss of each sample were measured using a $\mathrm{HV}$ capacitance bridge, and compared to theoretical models over the frequency range $10^{-5}$ to $10^{4} \mathrm{~Hz}$. The results indicate a real movement of charges/ions in the polymer bulk which causes a deterioration of the electrical insulation properties even in the absence of ac fields. The presence of a field further degrades the insulator. Samples were studied using the LIMM (laser-intensity-modulation method) to determine the distribution of polarization, assuming mean polarization is zero and space charge is absent. In the absence of the external field there is a presence of ionic charges of opposite polarity, which increases with increasing aging time and is highest at room temperature. The length of the twenty longest vented water trees was measured after various aging times, and it was found that trees grown at room temperature are longer than those grown at $65^{\circ} \mathrm{C}$. Also, the tree density was higher in specimens tested at room temperature.
\end{abstract}

\section{INTRODUCTION}

$\mathrm{P}$ OLYETHYLENE is an excellent electrical insulating material possessing low $\tan \delta$, good dimensional stability and solvent resistance. Both the cross-linked form XLPE (crosslinked polyethylene) and the LDPE (low density polyethylene) are semicrystalline. The folded regions of the macromolecule organize themselves in platelet forms to constitute the crystalline region whereas the amorphous regions, which lie between the crystallites, contain the chain ends and the interconnecting chains. The mechanical behavior of the polymer is mainly attributed to these amorphous regions. The dipole moment of the carbonhydrogen bond $(\mathrm{C}-\mathrm{H})$ of the polymer structure is quite low $(<0.3 \mathrm{De}-$ bye unit) and the material is generally regarded as hydrophobic until it is oxidized. XLPE absorbs $<100 \mathrm{ppm}$ of water at $20^{\circ} \mathrm{C}$. During the production impurities, water and residual cross-linking products (for XLPE) will be located in the amorphous regions. Both LDPE and XLPE experience aging when subjected to continued ac stress in humid environment which may lead to changes in dielectric and electrical properties and also to a formation of water trees.

The present paper reports the preliminary results of an investigation of the dielectric properties in the frequency range of $\sim 10^{-5}$ to $10^{4} \mathrm{~Hz}$, growth of water tree density and the spatial behavior of polarization in LDPE films aged in $0.1 \mathrm{M} \mathrm{NaCl}$ solution for a time period $\leqslant 454 \mathrm{~h}$ with an ac field of $6 \mathrm{kV} / \mathrm{mm}$ at $1 \mathrm{kHz}$ at room temperature and at $65^{\circ} \mathrm{C}$ by the LIMM.

\section{PREPARATION OF SAMPLES}

The specimens were prepared from Union Carbide HV grade crosslinked non-stabilized polyethylene (a base resin for the UC 4201 compound) in the form of flat sheets by molding in a hot press at $120^{\circ} \mathrm{C}$ and at $4.2 \mathrm{MPa}$ for $3 \mathrm{~min}$ to a thickness of $\sim 0.5 \mathrm{~mm}$. After molding, the flat sheets were removed from the press and allowed to cool to ambient 
temperature without employing any forced cooling. Subsequently, the sheets were cut into disks of $25.4 \mathrm{~mm}$ diameter, and the central portion of each disk was lightly sandblasted to accelerate initiation of vented water trees to within the first $2 \mathrm{~h}$ of voltage application. The diameter of the sandblasted area was $9 \mathrm{~mm}$.

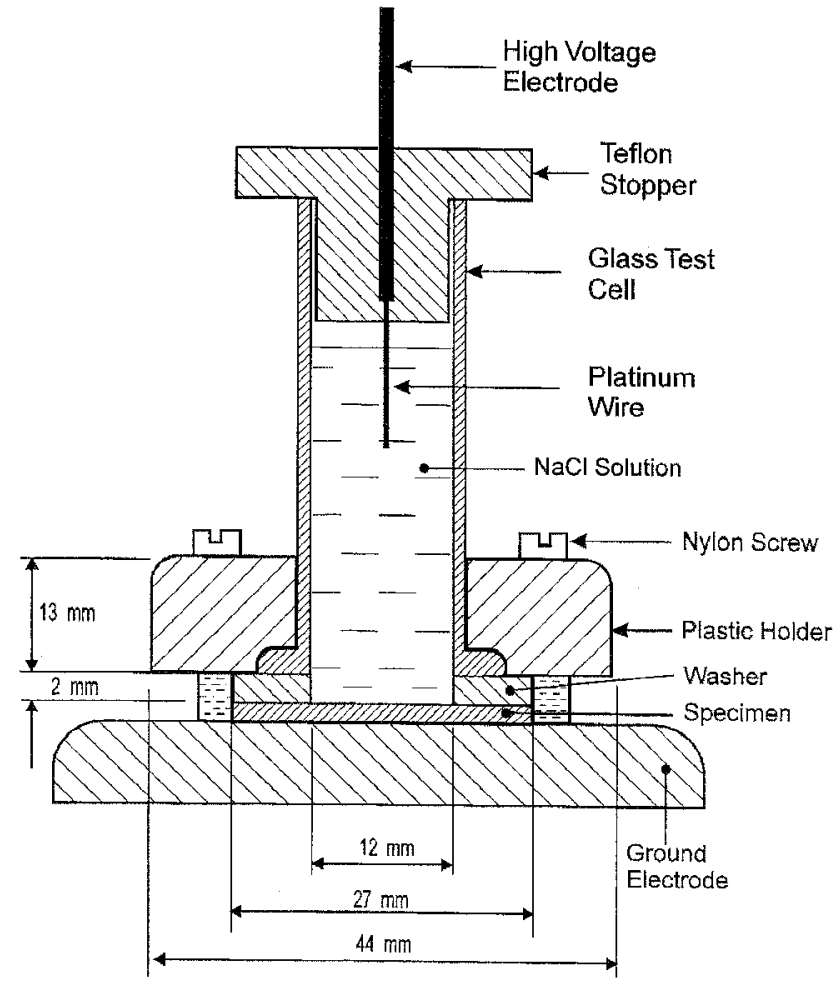

FIGURE 1. The test cell used to grow water trees.

The specimens were assembled in the test cells shown in Figure 1 . The sandblasted surface was always facing a liquid electrode connected to HV. The non-sandblasted surface, covered with a vacuum deposited aluminum electrode ( 15 to $22 \mathrm{~nm}$ thick), was connected to ground.

Specimens were subjected to a $1 \mathrm{kHz}$ ac stress of $6 \mathrm{kV} / \mathrm{mm}$ at an ambient temperature of $22 \pm 3^{\circ} \mathrm{C}$ and $65 \pm 1^{\circ} \mathrm{C}$ for $25,50,100,150,200$, 300 and $454 \mathrm{~h}$. A $0.1 \mathrm{M} \mathrm{NaCl}$ solution was used as the HV liquid electrode. An identical set of specimens were also aged in $0.1 \mathrm{M} \mathrm{NaCl}$ solution for the various aging times at both the ambient and at $65^{\circ} \mathrm{C}$ temperatures without any voltage. After each aging time, five voltage-aged specimens and one reference sample (immersed in ionic solution without voltage) were removed from the aging setup and one sample was dyed in methylene blue [1] to render water trees visible. Subsequently, the dyed specimens were microtomed into $125 \mu \mathrm{m}$ films, mounted on microscopic slides and examined for water tree length distribution using an optical microscope interfaced with the image analyzer. The remaining four undyed specimens were subjected to various dielectric tests as described below.

The capacitance and dielectric loss measurements at $\mathrm{HV}$ were performed using a current-comparator-based HV capacitance bridge developed by National Research Council of Canada [2]. The basic configuration of the bridge used in the measurements is shown in Figure 2. It consists of a current comparator with an adjustable ratio that compares the current from a standard $\mathrm{HV}$ low-loss gas-insulated capacitor $\mathrm{C}_{s}$ with

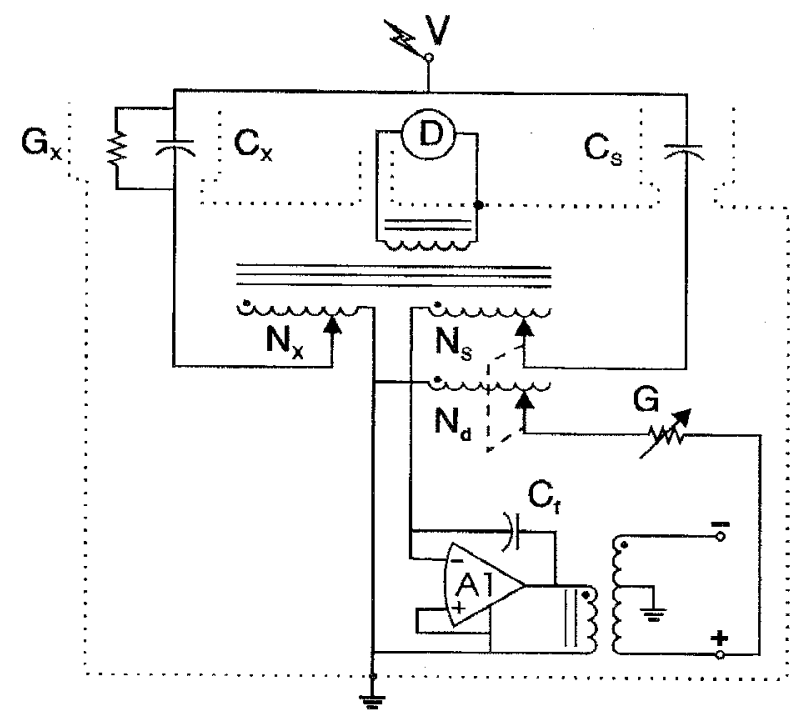

FIGURE 2. HV current-comparator-based capacitance bridge [12].

the current from a capacitor $\mathrm{C}_{x}$, to be measured. The ratio windings $\mathrm{N}_{s}$ and $\mathrm{N}_{x}$ [2] each have a nominal 1000 turns. The balance for the in-phase component (loss component) of the current from the unknown capacitor $C_{x}$ is obtained from an operational amplifier, $\mathrm{A} 1$ with a feedback capacitor $\mathrm{C}_{f}$ and an adjustable conductance, $\mathrm{G}$ connected to a winding, $\mathrm{N}_{d}$ having the same number of turns as the $\mathrm{N}_{s}$ winding. Mechanically coupling the switches that change the turns in windings $\mathrm{N}_{s}$ and $\mathrm{N}_{d}$ allows the bridge to read the dissipation factor directly. The bridge is balanced by adjustment of the $\mathrm{N}_{s}$ winding and the conductance $\mathrm{G}$. It has a resolution of $1 \mathrm{ppm}$ and is designed to detect a current unbalance of $4 \times 10^{-9}$ ampere-turns or $1 \mathrm{ppm}$ when $100 \mathrm{~V}, 60 \mathrm{~Hz}$ is applied to a $100 \mathrm{pF}$ standard capacitor. The uncertainty in the capacitance and dissipation factor measurements using the NRC bridge are estimated to be $\leqslant 0.02 \mathrm{pF}$ and $\leqslant 20 \mathrm{ppm}$, respectively.

The current comparator allows the bridge to achieve high measurement sensitivity without the need for a screen balance which is the case, for example, in bridges of the Schering type. Electromagnetic interference is eliminated by toroidal construction and magnetic shielding.

\section{DIELECTRIC PROPERTIES}

For the low frequency range the imaginary part of the complex permittivity $\varepsilon^{\prime \prime}$ was calculated from the desorption current $[3,4]$.

Figure 3 shows a typical behavior of the real $\chi^{\prime}$ and the imaginary $\chi^{\prime \prime}$ parts of the complex dielectric susceptibility $\chi$ of samples aged under different conditions, where $\chi^{\prime}$ and $\chi^{\prime \prime}$ are related to the real $\varepsilon^{\prime}$ and imaginary $\varepsilon^{\prime \prime}$ parts of the complex permittivity $\varepsilon$ for any chosen polarization.

$$
\begin{gathered}
\varepsilon^{\prime}(\omega)=\varepsilon_{\infty}+\varepsilon_{0} \chi^{\prime}(\omega) \\
\varepsilon^{\prime \prime}(\omega)=\varepsilon_{0} \chi^{\prime \prime}(\omega)
\end{gathered}
$$

$\varepsilon^{\prime \prime}(\omega)$ values (and hence $\chi^{\prime \prime}(\omega)$ values) were calculated from the isothermal discharge current using the following equation [5]

$$
\varepsilon^{\prime \prime}(\omega)=\frac{I(t)}{2 \pi f V C_{0}}=\frac{I(0.1 / f)}{2 \pi f V C_{0}}
$$

where $C_{0}$ is the geometric capacitance, $V$ the charging step voltage and $t=0.1 / f$ where $f$ is the Hamon frequency. When $\chi^{\prime \prime}(\omega)$ is known, 


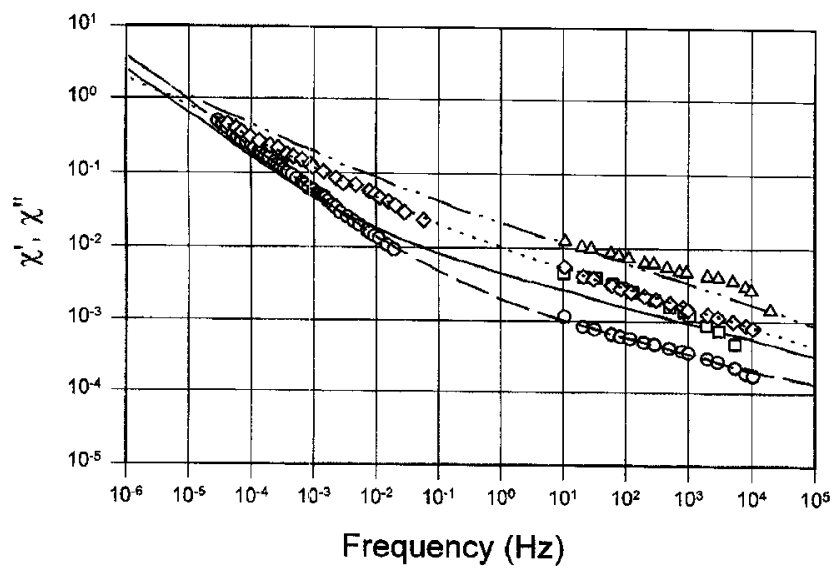

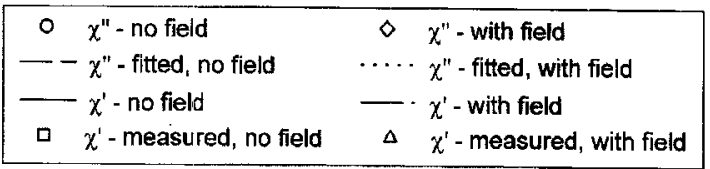

FIGURE 3. The behavior of real $\chi^{\prime}$ and imaginary $\chi^{\prime \prime}$ parts of the complex susceptibility of nonstabilized $\mathrm{PE}$ with aging in $\mathrm{NaCl}$ solution at room temperature with and without ac field $(6 \mathrm{kV} / \mathrm{mm})$ for $454 \mathrm{~h}$.

$\chi^{\prime}(\omega)$ can be calculated from

$$
\chi^{\prime}(\omega)=\frac{1}{\pi} \int_{-\infty}^{\infty} \frac{\chi^{\prime \prime}(x) d x}{x-\omega}
$$

where $\omega=2 \pi f$ is the angular frequency.

Numerical evaluation of the integral is possible using a routine from the NAG library [6]. It is an adaptive routine which employs a global acceptance criterion. Special care is taken to ensure that $\omega$ is never the end point of a subinterval. If $\omega$ is within a subinterval or close its end points, modified Clenshaw-Curtis integration of order 12 and 14 is performed. Otherwise, Gauss 7-point and Kronrod 15-point rules are used.

$\chi^{\prime \prime}(\omega)$ is assumed to be zero for frequencies larger than our largest measured frequency, and for lower frequencies $\chi^{\prime \prime}(\omega)$ is found from cubic-spline interpolation. Given a set of $\chi^{\prime \prime}(\omega)$ values, the corresponding values for $\chi^{\prime}(\omega)$ can thus be calculated easily, giving the continuous $\chi^{\prime}$ curves shown in Figure 3.

The measured and the calculated dielectric dispersion data have been fitted with the following expression for the high frequency region [7], (i.e. for $\omega>\omega_{p}$ ).

$$
\chi^{\prime \prime} \propto \frac{1}{\left(\frac{\omega}{\omega_{p}}\right)^{-m}+\left(\frac{\omega}{\omega_{p}}\right)^{1-n}}
$$

where $m$ and $n$ fall in the range $0<m<1,0<n<1$. $\omega_{p}$ is the frequency at which the maximum loss occurs in a typical relaxation process. It may be observed that for the high frequency part, $\chi^{\prime}$ and $\chi^{\prime \prime}$ maintain a constant frequency-independent ratio, i.e. in this $\log -\log$ representation (see Figure 3), $\chi^{\prime}(\boldsymbol{\omega})$ and $\chi^{\prime \prime}(\boldsymbol{\omega})$ are parallel above $\boldsymbol{\omega}_{p}$. This behavior represents an intra cluster carrier dominated system. The dielectric response at frequencies lower than $\omega_{p}$ indicates a presence of inter-cluster carrier-dominated system, i.e. a LFD (low frequency dispersion) or QDC (quasi dc) process in which the response rises steadily obeying a power law

$$
\chi^{\prime \prime}(\omega)=\cot \left(\frac{m \pi}{2}\right)\left[\chi(0)-\chi^{\prime}(\omega)\right] \propto \omega^{\prime \prime} \quad \omega<\omega_{p}
$$

The observed dielectric behavior (Figure 3 ) indicates $[7,8]$ a real movement of charges/ions in the polymer bulk (inter cluster motion) which causes a deterioration of the electrical insulation properties of the polymer in $\mathrm{NaCl}$ environment, even in the absence of ac fields, the effect of the presence of the latter being further degradation of the insulator.

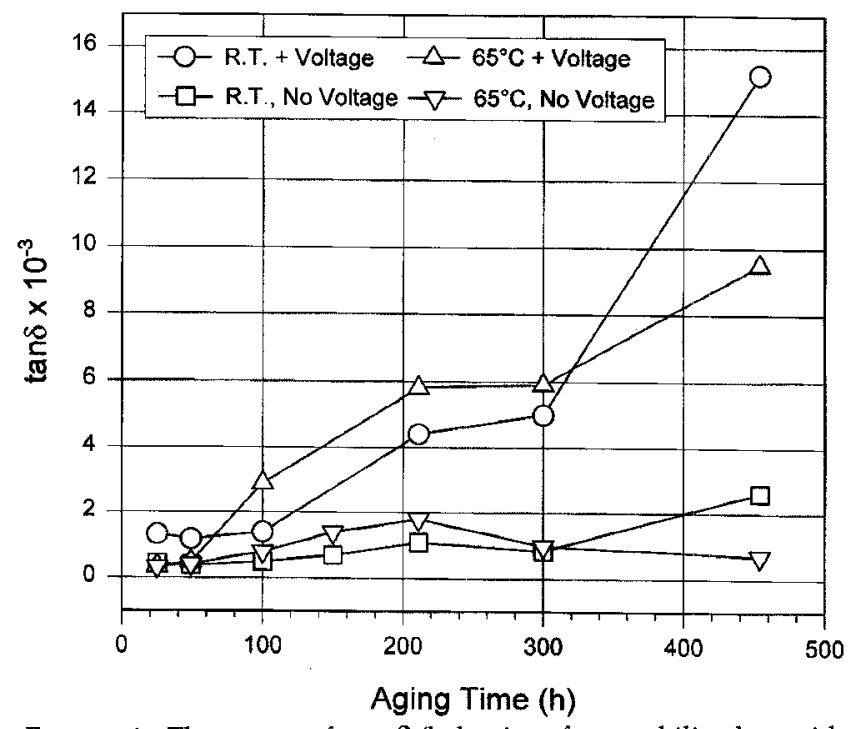

FIGURE 4. The nature of $\tan \delta$ (behavior of nonstabilized PE with aging time with and without ac field at room temperature and at 65 ${ }^{\circ} \mathrm{C}$ in $0.1 \mathrm{M} \mathrm{NaCl}$ solution.

The behavior of $\tan \delta$ (Figure 4) shows that it increases significantly with aging at room temperature in the presence of the ac field. The enhancement of $\tan \delta$ with ac field at $65^{\circ} \mathrm{C}$ is less than that at room temperature for an aging time of $454 \mathrm{~h}$. It appears that the $\tan \delta$ values are not significantly dependent on aging time and temperature in the absence of the field.

\section{EXPERIMENTAL SETUP FOR THE LIMM}

The LIMM employs a non-uniform sinusoidal thermal force in a wide frequency range which interacts with the spatially distributed polarization and space charge to produce complex sinusoidal pyroelectric current. This current is a unique function of the modulation frequency and the spatial distribution of polarization $[9,10]$. The experimental setup used for the LIMM experiments was basically the same as described in $[11,12]$.

The modulated thermal energy source was a He-Ne laser with a beam diameter of $\sim 2.0 \mathrm{~mm}$. The amplitude of the modulated radiation power was found to be $2 \mathrm{~mW}$, the mean value of the total intensity amounted to $3.5 \mathrm{~mW}$. LDPE samples (typical thickness $L=0.6 \mathrm{~mm}$ ) were blackened with a black marker resulting in an absorption layer of 1 to $2 \mu \mathrm{m}$ thickness which provided sufficient opaqueness (transmission $<1 \%)$ and high absorptivity $(>95 \%)$. The absorbed heat was assumed to be equal for both sides within an uncertainty of $<2 \%$, The remaining possible mismatch between the amplitude $I_{L}$ and $I_{Z}$ was neglected [13]. 
The LDPE samples were electrically connected and mechanically fixed between two brass rings. Two holes of $9.5 \mathrm{~mm}$ provided sufficiently good thermal insulation of the central irradiated area to the ambient, but also gave rise to thermoelastically generated bending resonances (flexural waves) as visible in several LiMM spectra.

The pyroelectric current was converted with an (inverting) current amplifier (Keithley, model 428) into an ac voltage, which was further processed by a digital signal processing lock-in amplifier (Stanford Research System, model 850). Note that the low impedance of the current amplifier $\left(Z<1 \mathrm{k} \Omega\right.$ at gain $\left.10^{8} \mathrm{~V} / \mathrm{A}\right)$ provides operation under dynamic short-circuit conditions.

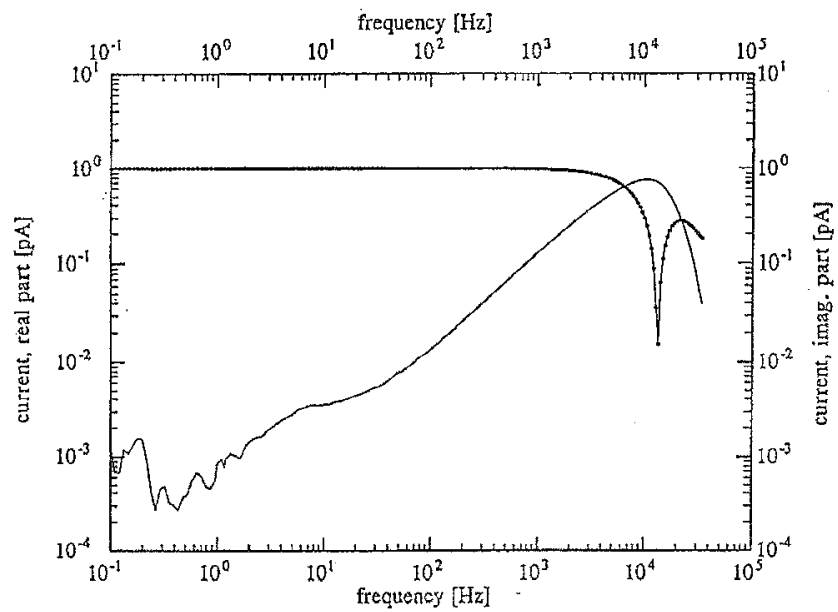

FIGURE 5. Gain/phase characteristics of the electronic and optical signal path in the LIMM, dotted curve: real part, plain curve: imaginary part.

In each experiment, 239 data points were acquired at logarithmically spaced frequencies from $0.1 \mathrm{~Hz}$ to $34 \mathrm{kHz}$. The gain-/phase characteristics of the electronic and optical signal path was determined with a fast photodiode and used for on-line data correction (Figure 5). A further correction step (dark measurement) was performed for each sample in order to eliminate high frequency interference between driving voltage and the input circuit.

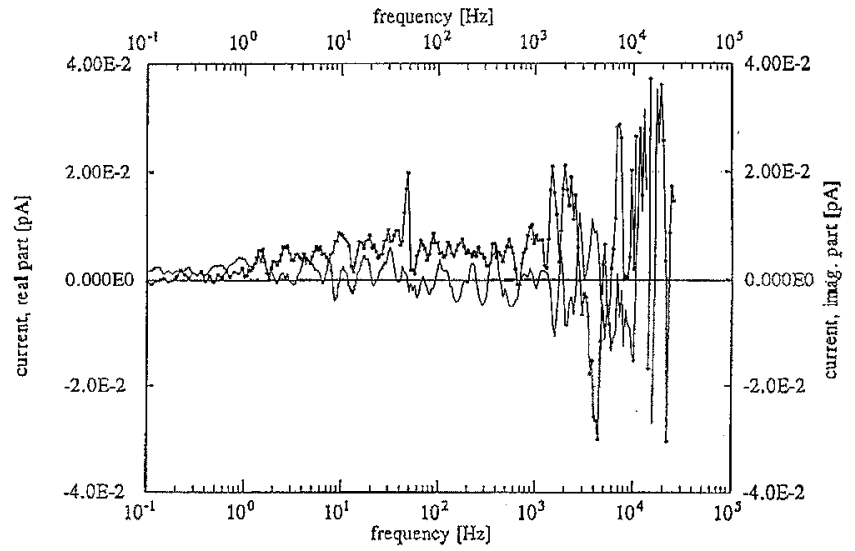

FIGURE 6. Typical current spectrum from LIMM (front side), dotted curve: real part, plain curve: imaginary part.

Pyroelectric currents were measured at a level of $\sim 3 \mathrm{fA}$ to $\sim$ $0.2 \mathrm{pA}$, which required noise rejection procedures like averaging (10X

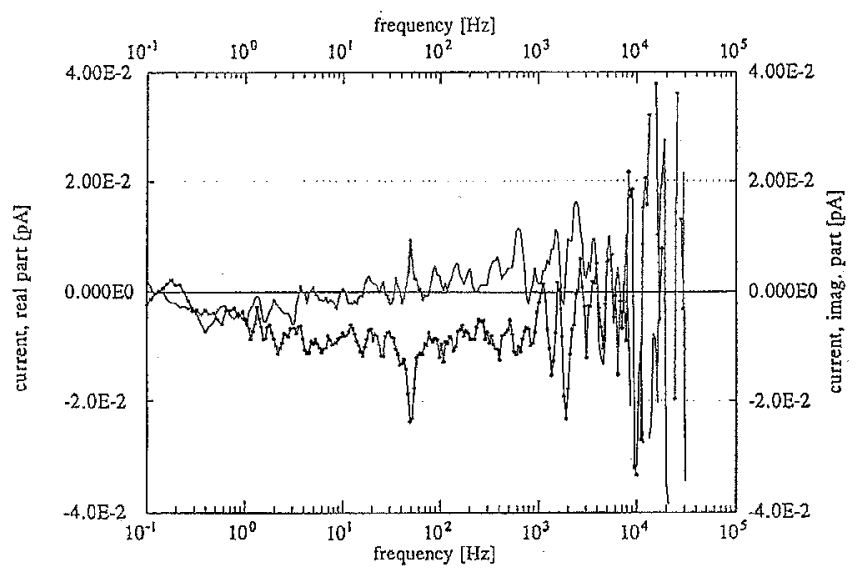

FIGURE 7. Typical current spectrum from LIMM (reverse side), dotted curve: real part, plain curve: imaginary part.

at each frequency) and long integration times. The measuring time for a complete spectrum was $\sim 20 \mathrm{~h}$, the typical noise level was found to be $7 \mathrm{fA}$ (standard deviation of the spectrum after subtraction of a polynomial fit curve of $10^{\text {th }}$ degree). The significance of small current values was verified with a dark measurement in the frequency interval below $100 \mathrm{~Hz}$ yielding a mean value of $<0.5 \mathrm{fA}$. Figures 6 and 7 show typical current spectra, one for each side of the specimen. A useful presentation of the measured data is the plot of the current $v s$. thermal diffusion length, Figure 8 . The strong increasing noise level at short diffusion lengths/high frequencies is mainly caused by the correction for the limited bandwidth of the current amplifier. Generally, data above $10 \mathrm{kHz}$ is not reliable, and has been excluded from the calculations.

\section{THEORY OF LIMM}

Radial heat transport becomes important when the thermal diffusion length $L_{D}$ is in the order of the beam size. From Figure 8 we see that even at the lowest frequency $L_{D} \sim 300 \mu \mathrm{m}$ is much smaller than the beam diameter $(2000 \mu \mathrm{m})$, and so the theory developed in [14] is valid.

It is well established that the inception and growth of water trees arise from electro-chemical oxidation, chain scission and formation of free radicals which are mostly ionic in nature. In this respect it may be argued that non-polar PE will have a non-uniform internal field arising from the existence of ionic charges due to progressive aging. However, when aging occurs at higher temperature, there will be enhanced mobility of such carriers leading to a neutralization of charges of opposite polarities together with a reduction of the non-uniform field gradient. In addition, it may be expected, assuning single carrier injection, that space charges with ac field will not be a dominant mechanism at room temperatures.

Furthermore, it has been shown $[15,16]$ that LDPE can have impurity dipole concentration of $1.6 \times 10^{27} \mathrm{~m}^{-3}$ which can be leached out by hexane treatment. Although the impurities may be leached out by chemical treatment, the concentration of trap sites will be unaltered, and on application of a high field, injected charges may be localized. However, it has been shown [17, 18] that on application of high fields subsequent to hexane treatment, the conductivity of PE is significantly reduced at least by two orders of magnitude, thus confirming that injection charges is not a dominant mechanism. 


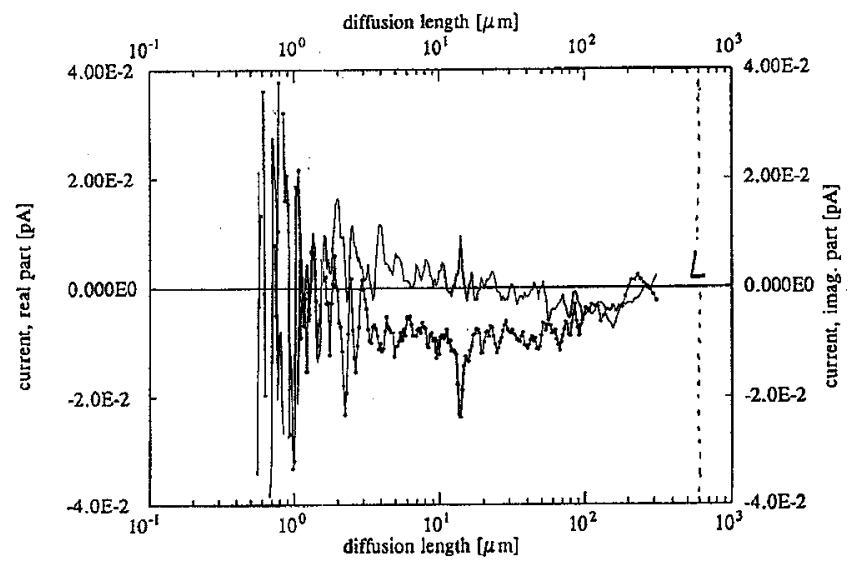

FIGURE 8. Typical current os. diffusion length plot, dotted curve: real part, plain curve: imaginary part.

The theory of LIMM shows that if the mean polarization is zero and charge is absent, the fundamental LIMM equations are [14]

and

$$
I_{L}=C \int_{0}^{1}\left[\frac{\sinh (v y)}{\sinh (v)}-y\right] \frac{d P^{*}}{d y} d y
$$

$$
I_{Z}=C \int_{0}^{1}\left[\frac{\sinh (v y)}{\sinh (v)}-(1-y)\right] \frac{d P^{*}}{d y} d y
$$

Here are $I_{L}$ and $I_{Z}$ are the pyroelectric current when the laser beam impinges on the surfaces, $x=L$ and $x=0$ respectively, $L$ being the sample thickness.

$$
\begin{gathered}
v=\left(\frac{\omega}{2 K}\right)^{1 / 2} L(1+j) \\
C=-\left(a_{p}+a_{x}+a_{\varepsilon}\right) A T_{0}
\end{gathered}
$$

and

$$
T_{0}=K q_{0} \frac{\eta}{k L}
$$

where $K$ is the thermal diffusivity of the sample, $a_{p}, a_{x}$ and $a_{\varepsilon}$ the relative temperature dependence of the polarization of the thermal expansion coefficient and the relative temperature dependency of the permittivity respectively, $\omega$ the angular frequency, $P^{*}$ the polarization, $y=x / L, \eta$ the absorptivity of the electrode (dimensionless), $k$ the thermal conductivity, $A$ the electroded area and $q_{0}$ the beam intensity. We define a new function

$$
\Phi=C \frac{d P^{*}}{d y}
$$

and it is this function which we calculated. The magnitude of the polarization can be recovered if all the values of $q_{0}, \eta, k, \alpha_{x}, \alpha_{e}$ are known. When $\Phi$ has been found, $P^{*}$ is calculated by numerical integration.

For numerical evaluation, the range of integration is divided into $m$ intervals of size $h=1 / m$ within which is $\Phi$ assumed to be constant $(m=40)$.

Then

$$
\begin{aligned}
I_{L} & =C \int_{0}^{1}\left[\frac{\sinh (v y)}{\sinh (v)}-y\right] \Phi d y \\
& \simeq \sum_{j=1}^{m} \Phi_{j}\left[\frac{\cosh (v y)}{v \sinh (v)}-\frac{y^{2}}{2}\right]_{y=\frac{(j-1)}{m}}^{y=\frac{j}{m}}
\end{aligned}
$$

and similarly

$$
I_{Z} \simeq \sum_{j=1}^{m} \Phi_{j}\left[\frac{\cosh (v y)}{v \sinh (v)}-\frac{y^{2}}{2}\right]_{y=1-\frac{j}{m}}^{y=1-\frac{(j-1)}{m}}
$$

The LIMM experiment is carried out for a series of frequencies $\omega_{i}$, $i=1,2, \ldots N$ for each of which there is a measured current $\left(I_{L}\right)_{\exp }$.

To match the experimental values to the model choose the set $\left\{\Phi_{j}\right\}$ which minimizes

$\sum_{f}\left\{\left[I_{L}-\left(I_{L}\right)_{e x p}\right]^{2}+\left[I_{Z}-\left(I_{Z}\right)_{e x p}\right]^{2}\right\}+\lambda \sum_{j=2}^{m}\left(\Phi_{j}-\Phi_{j-1}\right)^{2}$

$d P / d y_{j}$ is proportional to $\Phi_{j}$, so polarization $P$ can be found by numerical integration of the set $\left\{\phi_{j}\right\}$ using trapezoidal rule.

The last term in (15) is a smoothing term, and has to be chosen small but non-zero such that the computed solution does not contain any violent (numerically-induced) oscillations.

The set $\left\{\phi_{j}\right\}$ which minimizes expression (15) is calculated using a computer minimization routine from the NAG library [6]. Such a routine treats the members $\phi_{j}$ of the set as independent variables, and takes no account of the fact that the values $\phi_{j}$ are drawn from some continuous distribution. Because the data are noisy (Figures 6 and 7), this can result in a noisy (violently oscillating) solution with no physical meaning. By adding the $\lambda$ term in expression (15), the fit becomes worse but the solution becomes smoother as is increased. It is observed that over a wide range of values of $\lambda$, the shape of the solution (but not its magnitude) remains the same. In the present case, $L=0.6 \mathrm{~mm}$ and the diffusivity $K=1.74 \times 10^{-7} \mathrm{~m}^{2} \mathrm{~s}^{-1}$

Figure 9 shows the nature of deviation of the spatial polarization from the mean value of zero with aging. It may be observed that in the absence of the external ac field there is a presence of ionic charges of opposite polarities which increases with increasing aging time and its enhancement is highest at room temperature. At $65^{\circ} \mathrm{C}$ the deviation of spatial polarization from the mean value is insignificant in the presence of the aging field. It is possible that the external field at high temperature tends to make the internal space charge field more uniform in the bulk of the polymer and the enhanced conductivity will not provide a pyroelectric response. Further work is in progress to seek an explanation of this observed spatial polarization behavior.

\section{GROWTH OF WATER TREES}

$\mathrm{F}$ IGURE 10 shows the average length of the twenty longest vented water trees measured after various aging times. The trees grown at room temperature are longer than those grown at $65^{\circ} \mathrm{C}$. It was also observed that the tree density was higher in specimens tested at room temperature. This is illustrated in Figure 11 which shows microphotographs of water trees after $\sim 300 \mathrm{~h}$ of aging. Since after the same aging time, the water trees occupy a larger volume percentage of the specimens aged at room temperature than of those aged $65^{\circ} \mathrm{C}$, the overall water and ion content in the room temperature-aged insulation can be expected to be higher [19]. This can explain the larger values of loss tangent in room temperature-aged specimens. 


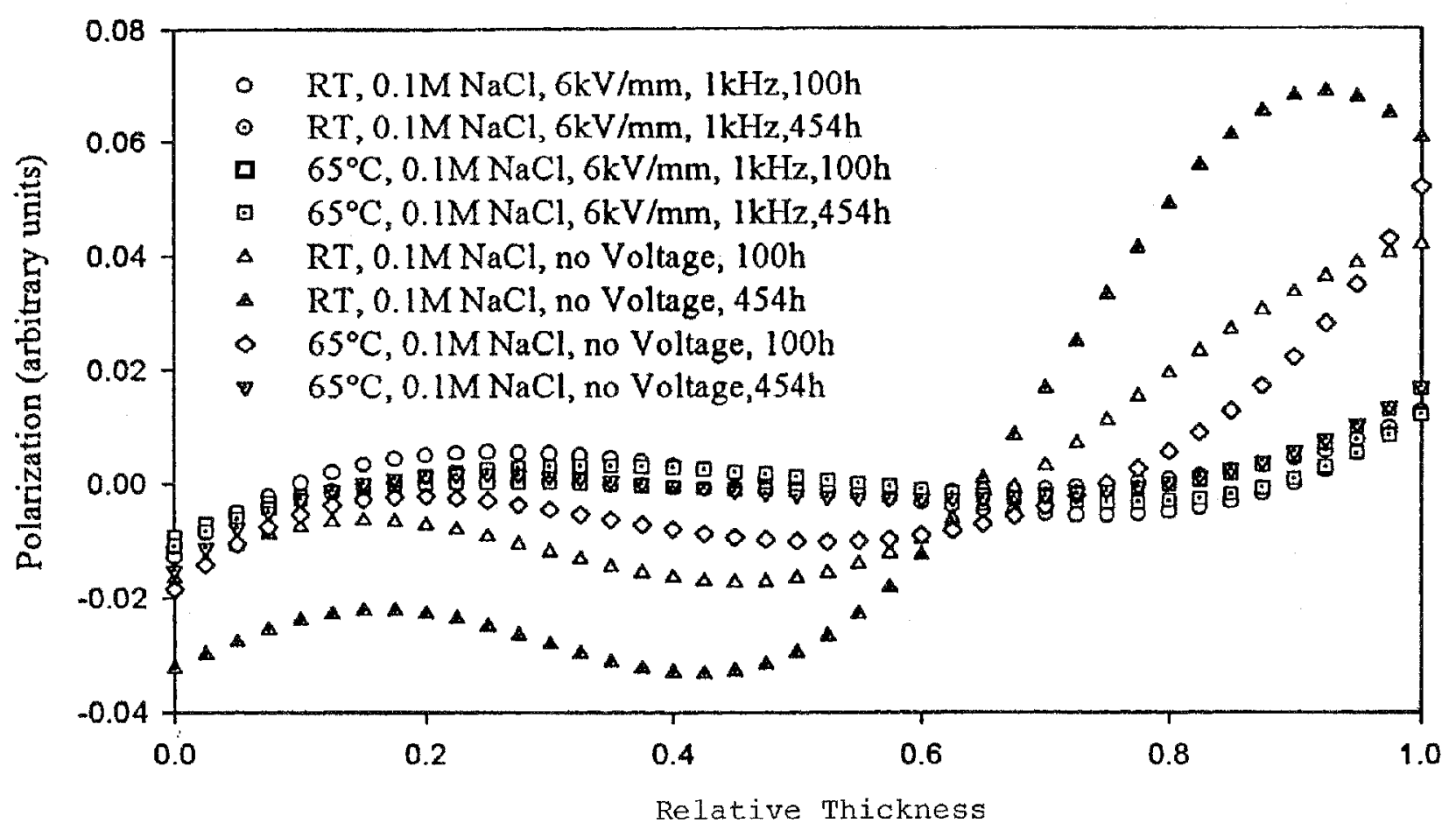

FIGURE 9. LIMM responses of nonstabilized polyethylene for different time periods with and without ac field $(6 \mathrm{kV} / \mathrm{mm})$ at $65^{\circ} \mathrm{C}$ and at room temperature.

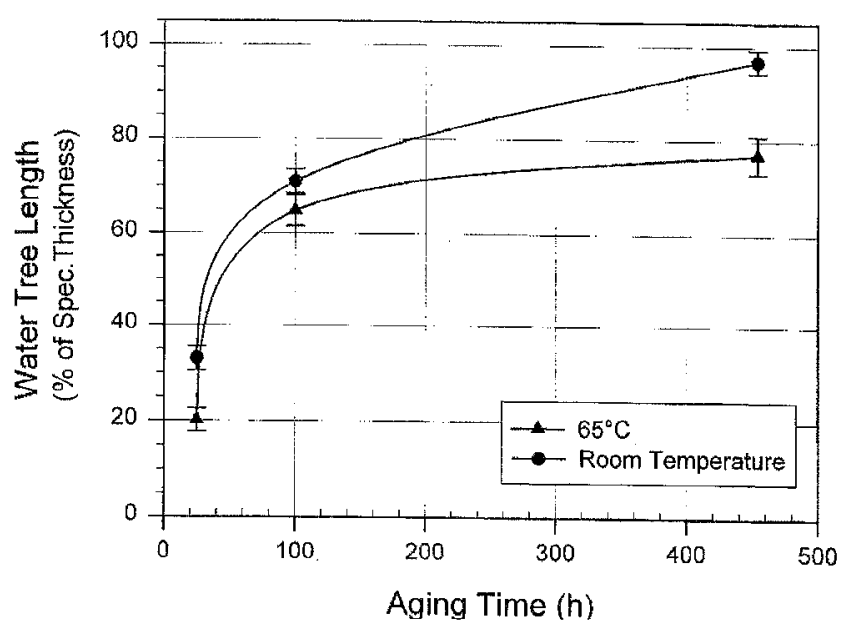

FIGURE 10. Average length of the twenty longest vented trees os. time of aging. Error bars indicate $95 \%$ confidence intervals.

\section{REFERENCES}

[1] A.C. Ashcraft and R. M. Eichhorn, "Method of Visualization of Water Trees by Staining", IEEE Trans. EI, Vol. 13, pp. 198-199, 1978.

[2] W. J. M. Moore and P. N. Miljanic, The Current Comparator, 1988 Peter Peregrinus Ltd., IEE Electrical Measurement Series 4, ISBN 0863411126.

[3] D. K. Das-Gupta and K. Joyner, "On the Nature of Absorption Current in Polyethylene Terephthalate (PET)", J. Phys. D:Appl. Phys., Vol. 9, pp. 829-840, 1976.

[4] D. K. Das-Gupta and R. S. Brockley, "Charging and Discharging currents in Polyvinylidenefluoride", J. Phys. D:Appl. Phys., Vol. 13, pp. 2101-2114, 1980.

[5] B. V. Hamon, "An Approximate Method for Deducing Dielectric Loss Factor from Direct-Current Measurements", Proc. IEE (London), Vol. 99, Pt IV, pp. 151-155, 1952.

[6] NAG Fortran Library Manual, NAG Ltd Oxford, UK, 1990.

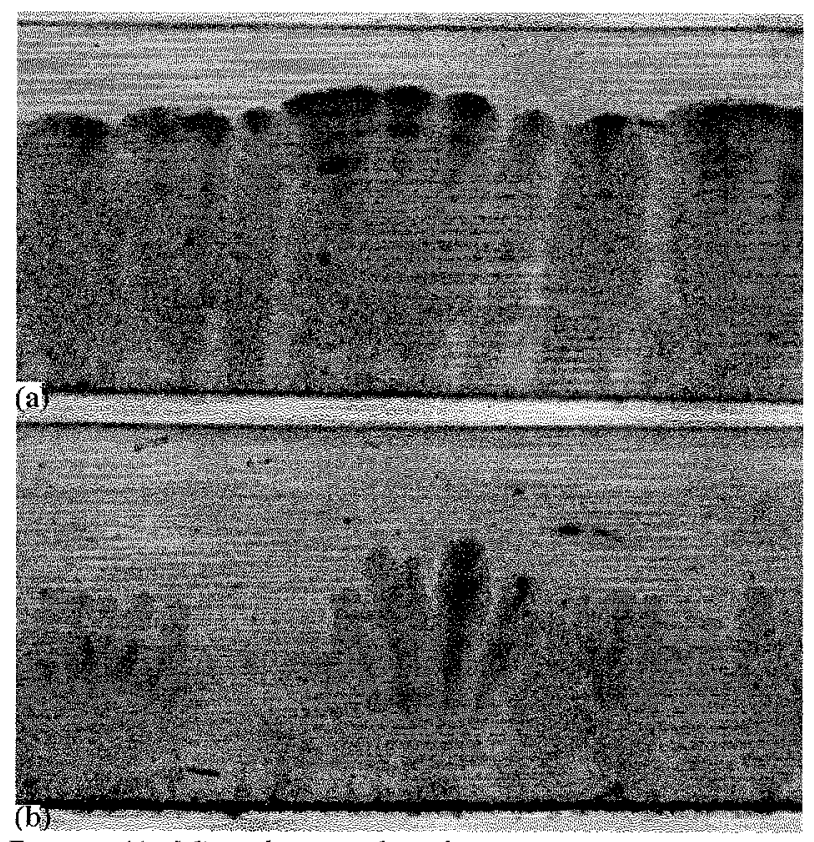

FIGURE 11. Microphotographs of water trees grown in nonstabilized polyethylene at room temperature (a) and $65^{\circ} \mathrm{C}$ (b). $\mathrm{Ag}$ ing conditions: $6 \mathrm{kV} / \mathrm{mm}, 1 \mathrm{kHz}, 0.1 \mathrm{M} \mathrm{NaCl}$ Solution, $300 \mathrm{~h}$.

[7] A. K. Jonscher, Dielectric Relaxation in Solids, Chelsea Dielectric Press, London, 1983.

[8] A. K. Jonscher, "The Universal Dielectric Response and its Physical Significance", IEEE Trans. EI, Vol. 27, pp. 407-423, 1992.

[9] S. B. Lang and D. K. Das-Gupta, "A New Technique for Determination of the Spatial Distribution of Polarization in Polymer Electrets", Ferroelectrics, Vol. 60, pp. 23-36, 1984. 
[10] S. B. Lang and D. K. Das-Gupta, "Laser Intensity Modulation Method: Technique for the Determination of Spatial Distribution of Polarization and Space Charge in Polymer Electrets", J. Appl. Phys., Vol. 59, pp. 2151-2160, 1986.

[11] F. Marlow, M. Wübbenhorst and J. Caro, "Pyroelectric Effect on Molecular Sieve Crystals Loaded with Dipole Molecules", J. Phys. Chem., Vol. 98, pp. 12315-12319, 1994.

[12] M. Wübbenhorst, J. van Turrhout and L. Alili, "Characterization of the Cure Epoxy by Pyroelectric Caloric Calorimetry", Ferroelectrics, Vol. 165, pp. 153-169, 1995.

[13] M. Wübbenhorst, $\mathrm{PhD}$ thesis, Spatial inhomogeneous polarization effect in polyvinylidene fluoride studied by means of the laser intensity modulation method, University of Leipzig, 1989.

[14] S. B. Lang, "Laser Intensity luodulation Method (LIMM) Experimental Techniques, Theory and Solution of the Integral Equation", Ferroelectrics, Vol. 118, pp. 343-361, 1991.

[15] R. W. Greaves, E. P. Fowler, A. Gooding and D. R. Lamb, "The Direct Piezoelectric Effect in Extruded Polyethylene", J. Mater. Sci., Vol. 9, pp. 1602-1608, 1974.
[16] D. K. Das-Gupta and J. S. Duffy, "Thermal Current Transients in Polyethylene", J. of Electrostatics, Vol. 14, pp. 99-109, 1983.

[17] D. K. Das-Gupta and M. K. Barbarez, "On Electronic Conduction in Polyethylene Films", J. Phys. D: Appl. Phys., Vol. 6, pp. 867-871, 1973.

[18] R. H. Partridge, "The Extrinsic Nature of Electrical Conductivity in Polyethylene", Polym. Lett., Vol. 5, pp. 205-208, 1967.

[19] B. Noirhomme, A. T. Bulinski, J.-P. Crine, R. J. Densley and S. S. Bamji, "Oxidation Phenomena in Water Treeing", EPRI report TR-102769, Palo Alto, 1993.

This manuscript is based on a paper given at the 9th International Symposium on Electrets, Shanghai, China, 25-27 September 1996.

Manuscript was received on 27 March 1997, in final form 21 October 1997. 